\title{
Distinct temporal hierarchies in membrane and cytoskeleton dynamics precede the morphological polarization of developing neurons
}

\author{
Annette Gärtner ${ }^{1, \pm, \S}$, Eugenio F. Fornasiero ${ }^{2, *, \ddagger}$, Flavia Valtorta ${ }^{2}$ and Carlos G. Dotti ${ }^{1,3, \S}$
}

\begin{abstract}
Final morphological polarization of neurons, with the development of a distinct axon and several dendrites, is preceded by phases where they have a non-polarized architecture. The earliest of these phases is that of the round neuron arising from the last mitosis. A second non-polarized stage corresponds to the bipolar neuron, with two morphologically identical neurites. Both phases have their distinctive relevance in the establishment of neuronal polarity. During the round cell stage, a decision is made as to where from the cell periphery a first neurite will form, thus creating the first sign of asymmetry. At the bipolar stage a decision is made as to which of the two neurites becomes the axon in neurons polarizing in vitro, and the leading edge in neurons in situ. In this study, we analysed cytoskeletal and membrane dynamics in cells at these two "prepolarity' stages. By means of time lapse imaging in dissociated hippocampal neurons and ex vivo cortical slices, we show that both stages are characterized by polarized intracellular arrangements. However, the stages have distinct temporal hierarchies: polarized actin dynamics marks the site of first polarization in round cells, whereas polarized membrane dynamics precedes asymmetric growth in the bipolar stage.
\end{abstract}

KEY WORDS: Axon, Polarity, Neuron, Migration, Membrane traffic, FRAP

\section{INTRODUCTION}

Neurons are extremely polarized cells, with axons specialized in delivering electrical signals that trigger the release of neurotransmitters from presynaptic contacts, and dendrites specialized in sensing neurotransmitters through specific receptors. This directional flow of information requires different molecular equipment and a distinct architecture of axons and dendrites. It remains an essential aspect in the neurosciences to understand how these specific features of axons are acquired during development.

${ }^{1}$ VIB Center for the Biology of Disease, KULeuven Center for Human Genetics, Leuven, Belgium and KULeuven, Department of Development and Regeneration 3000 Leuven, Belgium. ${ }^{2}$ S. Raffaele Scientific Institute/Vita-Salute University, Neuropsychopharmacology Unit, Division of Neuroscience, 20132 Milan, Italy.

${ }^{3}$ Centro de Biología Molecular Severo Ochoa, CSIC/UAM, 28049 Madrid, Spain.

*Present address: STED Microscopy of Synaptic Function, European

Neuroscience Institute, 37077 Göttingen, Germany.

‡These authors contributed equally to this work

§Authors for correspondence (Annette.Gaertner@cme.vib-kuleuven.be; cdotti@cbm.csic.es)

Received 15 January 2014; Accepted 1 August 2014
Neuronal polarization starts at the time of neuron generation, with the appearance of subtle molecular changes in the plasma membrane sufficient to warrant the growth of a first neurite (de Anda et al., 2005). We recently described that N-cadherin works as an initial important asymmetric tag, which determines the place from where the first neurite grows and to which the centrosome and the Golgi are directed in a second step (Gärtner et al., 2012; Pollarolo et al., 2011). The position of the second neurite is fixed at the opposite pole (de Anda et al., 2008; Menchón et al., 2011) by mechanisms that are still poorly characterized. This bipolar architecture supports migration (Hatanaka and Murakami, 2002; Sakakibara et al., 2014), as well as subsequent axonal and dendritic growth (Noctor et al., 2004), and thus influences the final organization of the entire brain.

The initially defined axonal identity is cell-autonomously regulated because isolated neurons in vitro can establish their first growth axis cell-autonomously, in the absence of asymmetric extracellular cues (Dotti et al., 1988; Powell et al., 1997). However, non cell-autonomous cues can impinge on those endogenous pathways and thus polarity establishment is a dynamic intrinsic-extrinsic process, where gradients of secreted molecules (e.g. reelin or Semaphorin 3A), extracellular matrix or cell-cell interactions influence neuronal positioning and axondendrite orientation, both in vivo and in vitro (Dimidschstein et al., 2013; Esch et al., 1999; Esch et al., 2000; Gärtner et al., 2012; Jossin and Cooper, 2011; Polleux et al., 1998; Polleux et al., 2000; Tissir and Goffinet, 2003).

Much is known about the regulation of axon growth from an already formed neurite (Polleux and Snider, 2010; Wiggin et al., 2005), but neuronal polarization begins before the appearance of the first neurite, and the polarity information must be transmitted throughout all different morphological stages in order to finally trigger polarized growth. How this is achieved has not been studied up to now, and thus we set out to describe in detail two morphologically symmetric stages, when the neuron is round and when the neuron develops two, oppositely located, neurites. Our results suggest that in round cells actin asymmetric dynamics has a predominant role in determining the site of first neurite formation; polarized membrane turnover in the bipolar neuron is a major predisposing factor for the late occurrence of polarized growth.

\section{RESULTS}

Intracellular dynamics in cultured hippocampal neurons at the round cell stage

Axon elongation is preceded and accompanied by the polarized flow of membrane cargoes (Bradke and Dotti, 1997; Bradke and Dotti, 1998; Flynn et al., 2009), by the polarized destabilization 
of F-actin in a single growth cone (Bradke and Dotti, 1999) and by the polarized stabilization of microtubuli (Neukirchen and Bradke, 2011; Witte et al., 2008). In light of these observations, we reasoned that similar polarized arrangements might precede the formation of the first neurite in post-mitotic neurons. This possibility was tested by time-lapse microscopy in developing neurons in vitro.

To analyse this 'pre-polarity stage' in vitro, freshly dissociated embryonic rat hippocampal neurons were transfected with GFPtagged membrane or cytoskeletal proteins immediately after cell dissociation. Transfected cells were kept in suspension for $2 \mathrm{~h}$ to avoid the initiation of differentiation before imaging, to provide time for the synthesis of the fluorescently tagged proteins and also to allow the regeneration of surface molecules which might have been cleaved during cell dissociation (Takeichi and Nakagawa, 2001). Cells were then transferred to poly-L-lysine (PLL)-coated coverslips and immediately imaged using confocal or wide-field microscopy, when still in the round morphology stage. These round neurons might be the in vitro correlate of round neurons in situ (see Attardo et al., 2008; Gärtner et al., 2012).

To visualize microtubule (MT) dynamics we used the neuronal specific plus-end-binding protein 3 (EB3, also known as MAPRE3). The time-lapse analysis revealed that MTs grow in a characteristic bipolar fashion, yet with the highest proportion of microtubules extending to one pole of the round cell (Fig. 1A).

Next, we analysed F-actin dynamics at the round neuron stage by transfecting cells with GFP-tagged utrophin cDNA (Utr-CH-GFP).
This F-actin probe is based on the calponin homology domain of utrophin (Utr-CH), which binds F-actin without stabilizing it (Burkel et al., 2007). Microscopy analysis revealed that filamentous actin was highly polarized to one pole of the round neuron (Fig. 1B).

To visualize the trafficking of newly synthesized membrane proteins, freshly dissociated neurons were transfected with the cDNA of GFP-tagged transferrin receptor (TfR-GFP), a marker for membrane carriers (Burack et al., 2000). This cargo is preferentially delivered to one pole of the round cell (Fig. 1C). The exocyst component Sec8 is also highly polarized in these round cells both in adhesion and in suspension (Fig. 1D), suggesting that the polarized trafficking of membrane is accompanied by polarized fusion.

In order to analyse endocytosis in round neurons, cells were incubated with the lipophilic dye FM4-64, which is taken up by endocytosis (Betz and Bewick, 1992). This experiment revealed the highly polarized distribution of endocytotic organelles (Fig. 1E). In agreement with this result, in neurons at this stage the endocytotic markers Rab5 and EEA1 also presented a polarized distribution (Fig. 1E; supplementary material Fig. $\mathrm{S} 1 \mathrm{C})$.

We next sought to define whether any of these polarized events temporally correlate with the first sign of morphological polarization, that is, the growth of the first neurite. In previous works we showed that the Golgi and the centrosome/microtubuleorganizing centre (MTOC) are highly motile before polarization and become fixed at the base of the first neurite after this has
A EB3-GFP
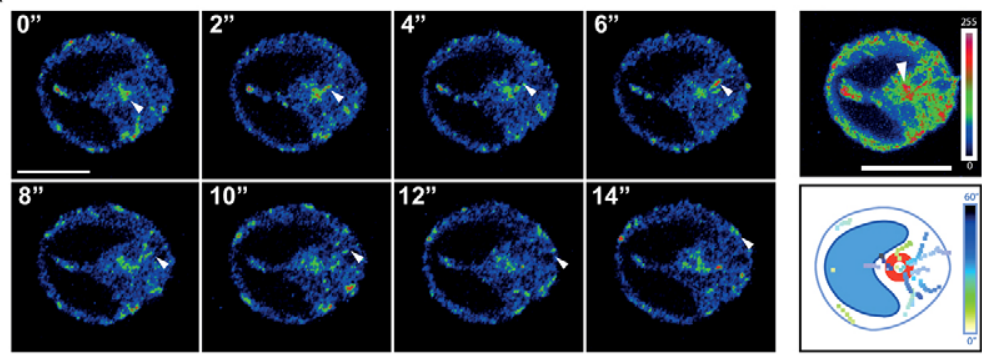

B Utr-CH-GFP
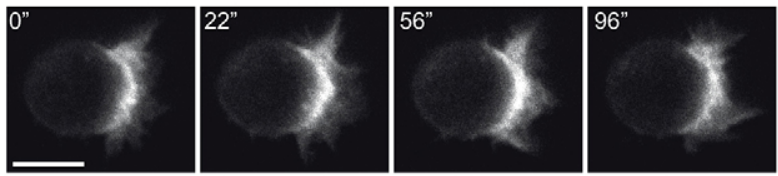

C TfR-GFP

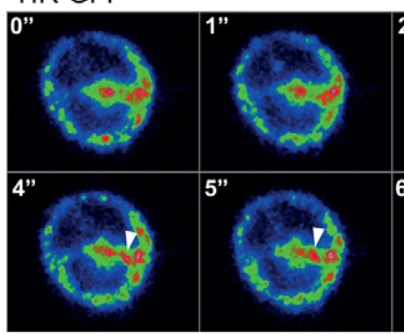

D

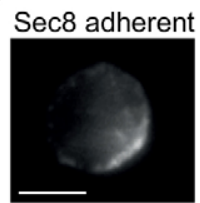

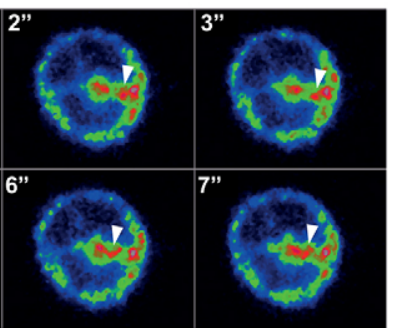

E

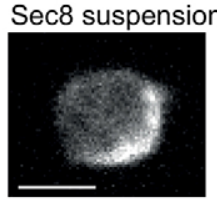

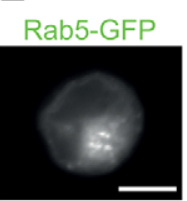

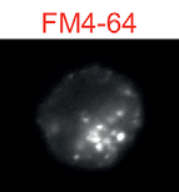

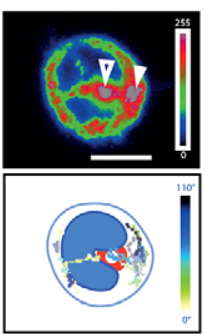

merge

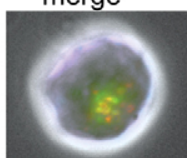

Fig. 1. Round neurons are polarized. (A-C) Time-lapse imaging of hippocampal neurons that were transfected with constructs encoding chimaeras of GFP and proteins labelling different intracellular compartments. Neurons were imaged for the indicated time immediately after plating. (A) Tracking of MT plus-ends in neurons transfected with EB3-GFP. Left panels: arrowheads indicate single plus-ends tracked over time. Upper right panel: maximal intensity projection of all time points. The arrowhead points to the Golgi complex. MTs preferentially, but not exclusively, extend to the pole of the cell closest to the MTOC, as shown with a rainbow red-green-blue (RGB) lookup table (LUT). Lower right panel: tracking of several plus-ends over $60 \mathrm{~s}$ (inverted green-fire-blue LUT; $n=26$ cells, 3 not polarized). (B) In vivo imaging of $\mathrm{F}$-actin in neurons transfected with Utr-CH-GFP. F-actin is polarized at one pole and appears very dynamic (images representative of $n=15$ cells).

(C) Membrane trafficking visualized in neurons transfected with TfR-GFP. Left panels: arrowheads indicate single carriers tracked over time. Upper right panel: maximal intensity projection of all time points. The arrowhead points to the Golgi complex (RGB LUT). Lower right panel: displays tracking of several carriers over $110 \mathrm{~s}$ (inverted green-fire-blue LUT, $n=10$ cells, all polarized). (D) Distribution of the exocyst component Sec8 as visualized by immunolabelling in round neurons either adhered to PLL (left panel, $n=38$ cells, quantification in supplementary material Fig. S1) or in suspension (right panel, $n=25$ cells, quantification in supplementary material Fig. S1). (E) Neurons transfected with a Rab5-GFP-expressing construct and loaded for 1 min with FM4-64. Both endocytic markers reveal a polarized distribution of endocytic vesicles ( $n=4$ cells, quantification of endocytic markers in supplementary material Fig. S1). Scale bars: $5 \mu \mathrm{m}$. 
formed (Gärtner et al., 2012). We therefore hypothesized that before the emergence of the first neurite, the polarity of cytoskeleton dynamics and membrane trafficking might reorganize their spatial orientation with time. In order to test this hypothesis, we transfected a Golgi marker (GT-TAGRFP) together with fluorescent reporters to mark microtubule dynamics (EB3-GFP), F-actin dynamics (Utr-CH-GFP) or membranedirected traffic (TfR-GFP). Alternatively, we directly marked the Golgi with wheat germ agglutinin (WGA). This study revealed that MTs and membrane trafficking undergo slow and steady rotational movements before morphological polarization together with the Golgi (Fig. 2A,B). This co-positioning is also evident for the Sec8 compartment and endosomes (supplementary material Fig. S1). F-actin condensations on the cell cortex also changed position before any sign of morphological polarity was evident, yet the position of actin clusters did not spatially match with the position of the Golgi (Fig. 2C). The first neurite formed at the place where $\mathrm{F}$-actin condensed and remained at a fixed position.
Only when the first neurite became evident did the Golgi complex started to reorient towards this site (Fig. 2D). This was also the case for microtubule growth (Fig. 2E), transport of new membrane (Fig. 2F), localization of the exocyst (supplementary material Fig. S2A) and endocytosis (supplementary material Fig. S2B).

We recently demonstrated that $\mathrm{N}$-cadherin condensation at one pole precedes the outgrowth of the first neurite (Gärtner et al., 2012). Thus, we speculated that $\mathrm{N}$-cadherin clustering is responsible for the restriction of actin dynamics to one pole. In order to test this possibility, we analysed in parallel $\mathrm{N}$-cadherin and F-actin localization in round neurons. For this, neurons transfected with the F-actin indicator Utr-CH-GFP were incubated with latex microspheres coated with the extracellular $\mathrm{N}$-cadherin domain. Those beads were used in order to detect sites of high surface expression of $\mathrm{N}$-cadherin in developing neurons, whereas tenascin-C-labelled beads served as control for $\mathrm{N}$-cadherin specificity. Time-lapse analysis demonstrated that

A
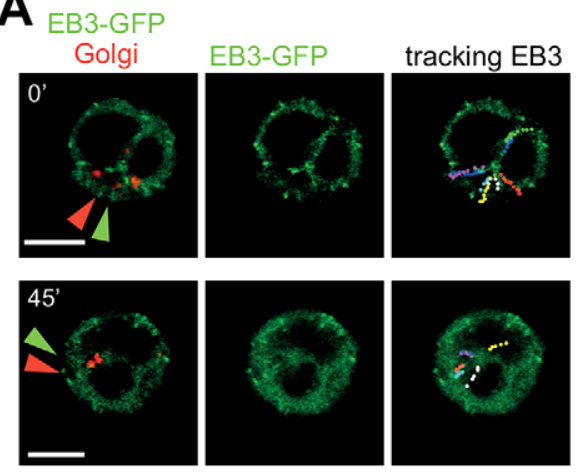

B
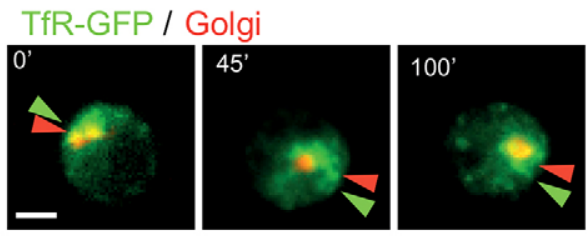

C Utr-CH-GFP / Golgi
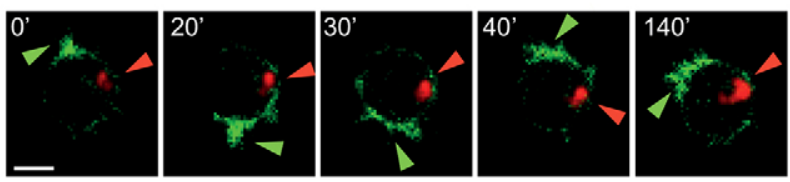

\section{Utr-CH-GFP / Golgi}

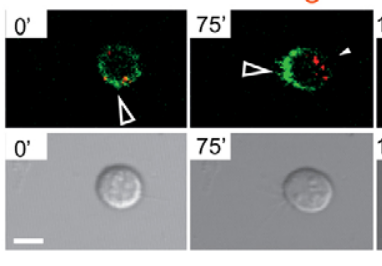

E

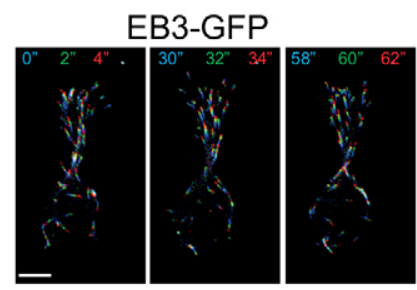

$\mathbf{F}$
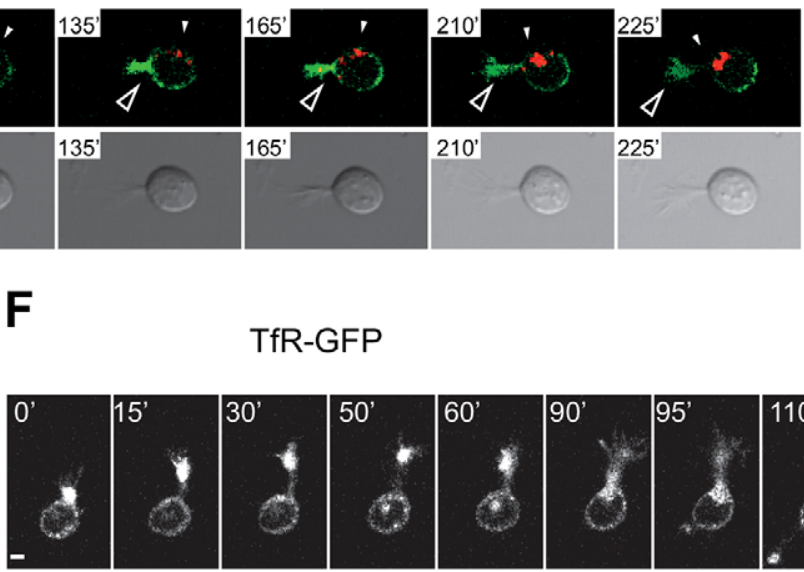

TfR-GFP
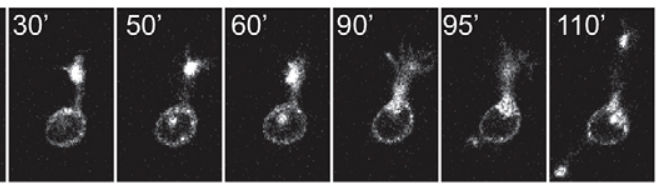

Fig. 2. In round neurons, polarity is not yet stable and becomes stabilized with the growth of the first neurite. (A-D) Time-lapse imaging of hippocampal neurons transfected with constructs encoding the same GFP chimeras shown in Fig. 1 and with a probe labelling the Golgi complex (GT-TAGRFP in B and C, WGA conjugated to Alexa Fluor 647 in A and D). Neurons were imaged for the indicated time immediately after plating. (A) Tracking of MT plus-ends in neurons transfected with EB3-GFP at two different time points $(0$ and $45 \mathrm{~min})$ in 1 -s frames. Tracking of EB3 tips is shown in the right panel. Red arrowheads, Golgi complex; green arrowheads: polarized orientation of MTs (images representative of $n=15$ cells). (B) Membrane trafficking visualized in neurons transfected with TfR-GFP. Red arrowheads, Golgi complex; green arrowheads, polarized orientation of TfR-GFP ( $n=13$ cells). (C) In vivo imaging of F-actin in neurons transfected with Utr-CH-GFP. Red arrowheads, Golgi complex; green arrowheads, polarized orientation of F-actin condensation ( $n=29$ cells).

(D) Neurons transfected with Utr-CH-GFP in order to visualize F-actin in vivo and incubated with Alexa-Fluor-647-conjugated WGA in order to show the position of the Golgi. Neurons were imaged for the indicated time after plating. Open arrowheads, the site of maximal F-actin condensation shifts with time and is stabilized in one place as soon as the first neurite bud starts to extend; the Golgi complex (red, indicated by white filled arrowhead) is successively positioned towards the first neurite ( $n=9$ cells). (E) Neurons transfected with EB3-GFP for tracking MT plus-ends. MT plus-ends are growing towards the first bud periphery, as seen from the overlay of three subsequent time frames (shown in blue, green and red, as indicated). (F) Neurons transfected with TfR-GFP in order to follow transport to the plasma membrane. Carriers are always transported into the first neurite. Scale bars: $5 \mu \mathrm{m}$. 
recruitment and stabilization of F-actin occurs at, or in the close proximity to, the $\mathrm{N}$-cadherin bead accumulation (Fig. 3A). To quantify the colocalization, transfected neurons were fixed $2-4 \mathrm{~h}$ after incubation with the coated beads and analysed for the location of the $\mathrm{N}$-cadherin or tensacin $\mathrm{C}$ beads with respect to the $\mathrm{F}$-actin condensation. This analysis revealed that, in the majority of cases, F-actin fluorescence peaked in close proximity to the $\mathrm{N}$ cadherin bead (see frequency distribution of the angle between the centre of the $\mathrm{N}$-cadherin-coated bead and the maximum of the cortical Utr-CH-GFP fluorescence in Fig. 3B), but was independent on the localization of tenascin-C-coated beads. Overall, these data indicate that adhesion-mediated restriction of F-actin dynamics in the round neuron marks the place of membrane and microtubule polarization and prepares neurons for their first morphological polarization.

\section{Intracellular dynamics in cultured hippocampal neurons at the bipolar cell stage}

We have previously demonstrated that in newly formed hippocampal neurons in culture, the first neurite has the highest chance to become the axon. We also showed that the second neurite forms at the opposite pole (de Anda et al., 2008; Menchón et al., 2011). Here, we set out to study the mechanisms by which the first neurite eventually acquires specific growth characteristics and whether or not these mechanisms are conserved in vitro and in vivo. To tackle this issue we repeated the same series of intracellular dynamics experiments as above for bipolar neurons (Fig. 4).

We have previously demonstrated that in vitro the axon usually forms from the neurite closest to the centrosome and Golgi (de Anda et al., 2008). Moreover, we have shown that the Golgi moves to the vicinity of the first neurite. Hence, knowing the position of the Golgi or centrosome-MTOC allowed us to predict which of the two neurites of the bipolar neuron in vitro would later become the axon. First, we compared the dynamics of MTs in the two neurites of bipolar neurons using a GFP-tagged $\alpha$-tubulin fusion protein ( $\alpha$-tub-GFP). We photobleached both of the two neurites and measured the time for recovery of $\alpha$-tubGFP. This experiment revealed identical tubulin turnover in both neurites (Fig. 4A). To control that our assay measured real MT turnover and not just the diffusion of $\alpha$-tubulin monomers, we repeated the experiment in the presence of the MT-stabilizing drug taxol. As expected, there was nearly no recovery of MTs in the presence of taxol, indicating that we were determining bona fide MT turnover rates (Fig. 4A). To strengthen this conclusion, we performed fluorescence recovery after photobleaching (FRAP) experiments in cells transfected with either EB3-GFP or YFP-doublecortin (DCX-YFP), a MT-binding protein normally required for neuronal migration. Once more, the recovery rates in both neurites were very similar (Fig. 4A). These results show that even though MTs originate from the asymmetrically positioned centrosome, MT dynamics are not asymmetric (polarized) in bipolar neurons. We also counted the number of EB3 comets in the two neurites after live imaging experiments of EB3-GFP, in order to determine the proportion of MT ends in the two neurites. This measurement did not reveal any differences in the two neurites (Fig. 4B).

In order to compare the dynamics of F-actin in the two neurites of bipolar neurons, we performed FRAP experiments on cells expressing the chimeric fusion protein actin-GFP. Photobleaching followed by analysis of the time for recovery revealed a similar turnover in both neurites (Fig. 4C). In order to ensure that our analysis reflected F-actin turnover rate and not the diffusion of actin monomers, we repeated the experiment using Utr-CH-GFP. Again, the recovery rates were comparable in both neurites (Fig. 4C). As a further control, FRAP analysis was performed in cells treated with the actin-stabilizing drug jasplakinolide. This experiment revealed a similar decreased recovery of fluorescence in both neurites (Fig. 4C).

To analyse membrane dynamics in the two neurites of bipolar neurons, we transfected cultured hippocampal neurons with TfRGFP and allowed the newly synthesized protein to undergo

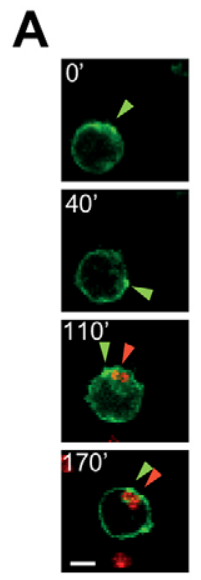

B

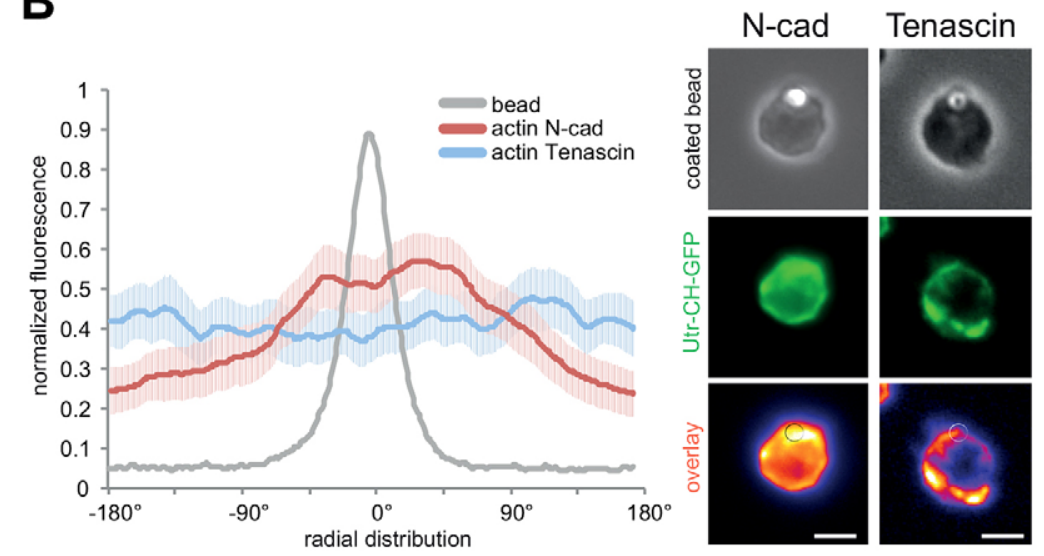

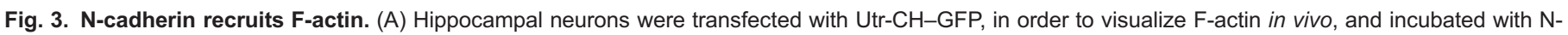
cadherin-coated latex microspheres before imaging the recruitment of F-actin. The N-cadherin-coated microsphere(s), which contacts the neuron between

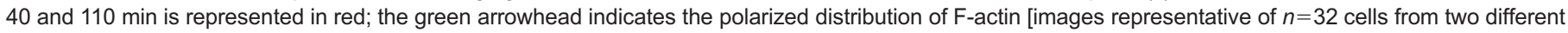

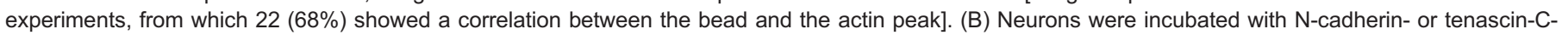
coated latex microspheres and fixed after 2-4 h. F-actin was visualized using Utr-CH-GFP or phalloidin. Left panel: radial distribution of the mean \pm s.d. fluorescent signal of actin measured in fixed neurons contacting either a $\mathrm{N}$-cadherin- or a tenascin-coated bead. For each cell, the fluorescence signal is normalized to the maximum peak, aligned to the localization of the bead $\left(0^{\circ}\right)$ and averaged ( $\mathrm{N}$-cadherin beads, $n=63$ cells from three different experiments;

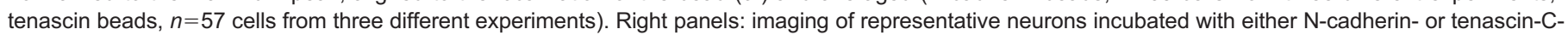
coated latex microspheres. Scale bars: $5 \mu \mathrm{m}$. 

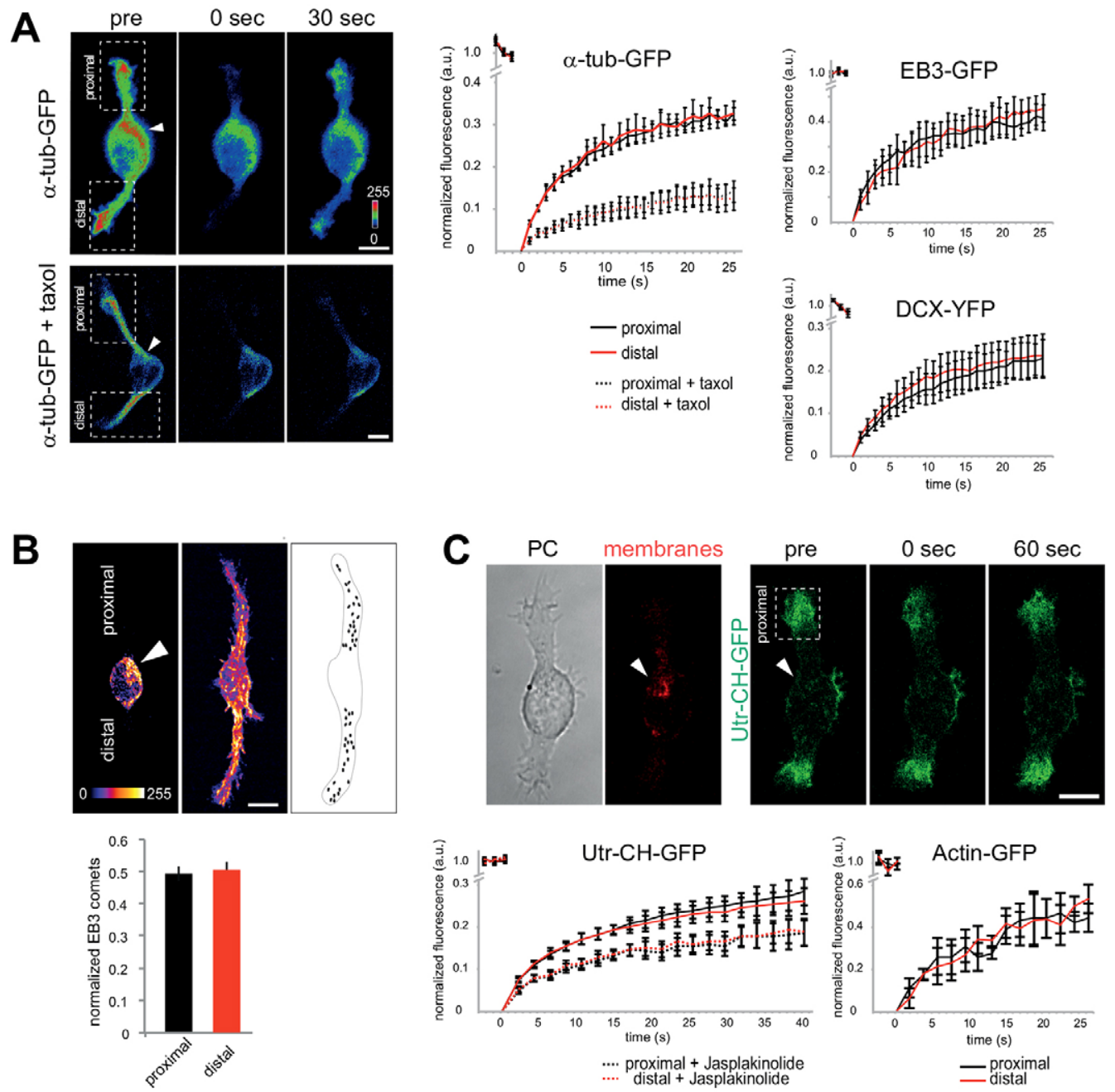

Fig. 4. The microtubule and the actin cytoskeleton do not show dynamic polarization in neurites of bipolar neurons either in vitro or in situ. Live confocal imaging and corresponding FRAP analysis of cultured hippocampal neurons at the bipolar stage (0 DIV). GFP-tagged proteins are represented by use of a red-green-blue (RGB) (A) or fire lookup table (LUT) (B), or are in green (C), and label either MTs [ $\alpha$-tubulin-GFP, EB3-GFP or Dcx-YFP (A,B)] or F-actin [Utr-CH-GFP; C]. Following selective photobleaching (dashed white box) of the proximal and/or the distal neurite fluorescence recovery was monitored for 25-60 s. (A) Representative examples of MT photobleaching experiments in neurons expressing $\alpha$-tubulin-GFP (left images) and quantifications for $\alpha$ tubulin-GFP, EB3-GFP and Dcx-YFP (right graphs). Bleaching was carried out simultaneously in both neurites. Taxol (10 nM) was added as a control in $\alpha-$ tubulin-GFP-expressing neurons to reduce MT dynamics. (B) Representative confocal pictures and quantification of EB3-GFP clusters (comets) in transfected neurons at the bipolar stage. Left panel: confocal image of the cell soma with the MTOC clearly visible. Middle left: confocal image in the neurite focal plane. Middle and right panels: EB3 comets were counted in live imaged neurons following $30 \mathrm{~s}$ movies in the neurite focal plane with the manual tracking tool of Image J. The outline of the cell and the detected EB3 clusters (black dots) are shown. Lower panel: mean percentage of EB3 clusters ( \pm s.e.m.) distributed in either neurite from three independent experiments (>10 cells per experiment). (C) F-actin photobleaching was performed in one neurite at a time (randomly proximal or distal) to minimize cellular stress. Only the bleaching of the proximal neurite is shown; plots represent recovery from both neurites. Phase contrast $(\mathrm{PC})$ is shown to outline the entire cell. The proximal and the distal neurite with respect to the Golgi complex have similar recovery rates in all conditions analysed. Mean \pm s.e.m. recovery curves are shown (for all conditions $n>30$ cells, from four independent experiments). Data were analysed by Student's $t$-test. Scale bars: $5 \mu \mathrm{m}$. White arrowheads, Golgi and centrosome position.

transport to both neurites. We then photobleached TfR-GFP in each neurite and measured the entry of membrane carriers from the cell body into the photobleached region. This assay revealed a large difference between the recovery rates of the two neurites, showing a significantly faster membrane supply into the Golgiproximal neurite (Fig. 5A,B). We obtained consistent results by analysing the transport of generic or bulk membrane carriers visualized with a palmitoylated GFP (Fig. 5C) and the transport of the ER-Golgi intermediate compartments (ERGICs) labelled with YFP-tagged p58 (LMAN1) (Fig. 5D; Sannerud et al., 2006). We repeated this analysis using another membrane marker, the TrkB receptor, a protein involved in axonal growth stimulated by brain-derived neurotrophic factor (BDNF) (Shelly et al., 2007). Again, we found a faster recovery of TrkB-GFP fluorescence in the Golgi-proximal neurite (Fig. 5E). Interestingly, endocytosis, as measured by uptake of the styryl dye FM4-64 was also more prominent in the Golgi-proximal neurite (Fig. 5F). Taken together, these results indicate that polarized exo-endocytosis predisposes the Golgi-proximal neurite to have different polarized growth.

\section{Intracellular dynamics in bipolar neurons in situ}

The previous data show that membrane turnover in bipolar neurons in vitro is intrinsically polarized, whereas actin and MT 
A

pre-bleaching bleaching
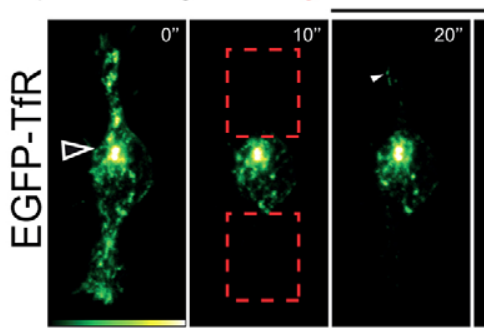

recovery

B

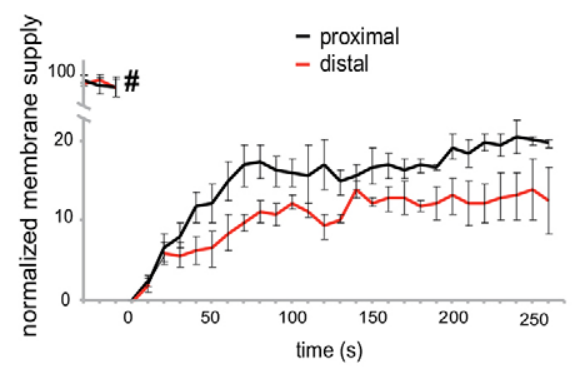

C

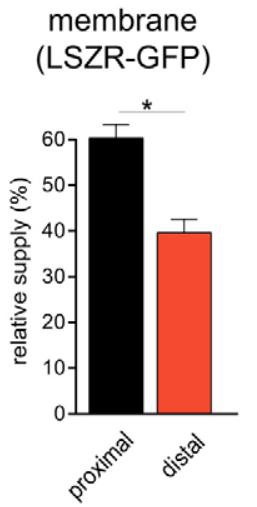

\section{ERGIC} (p58-YFP)

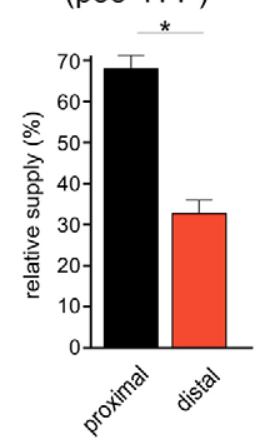

E
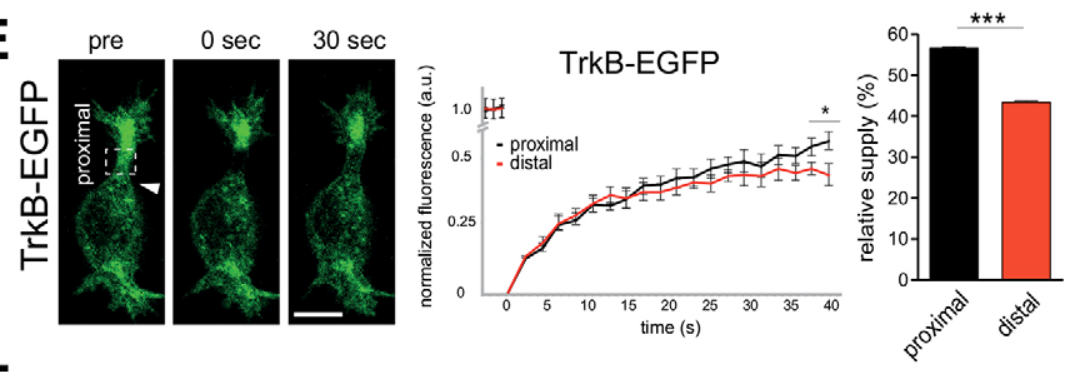

$\mathbf{F}$
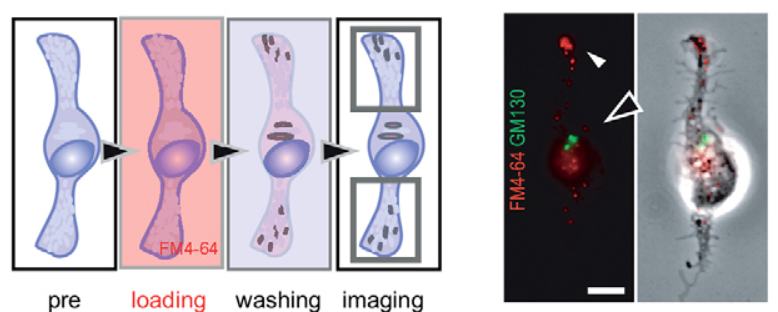

pre loading washing imaging
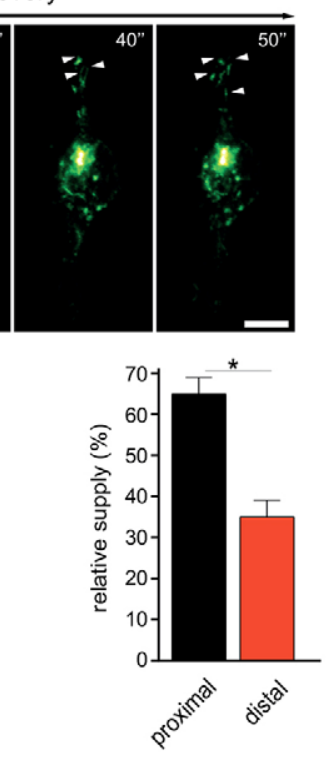

$+2$ 
A
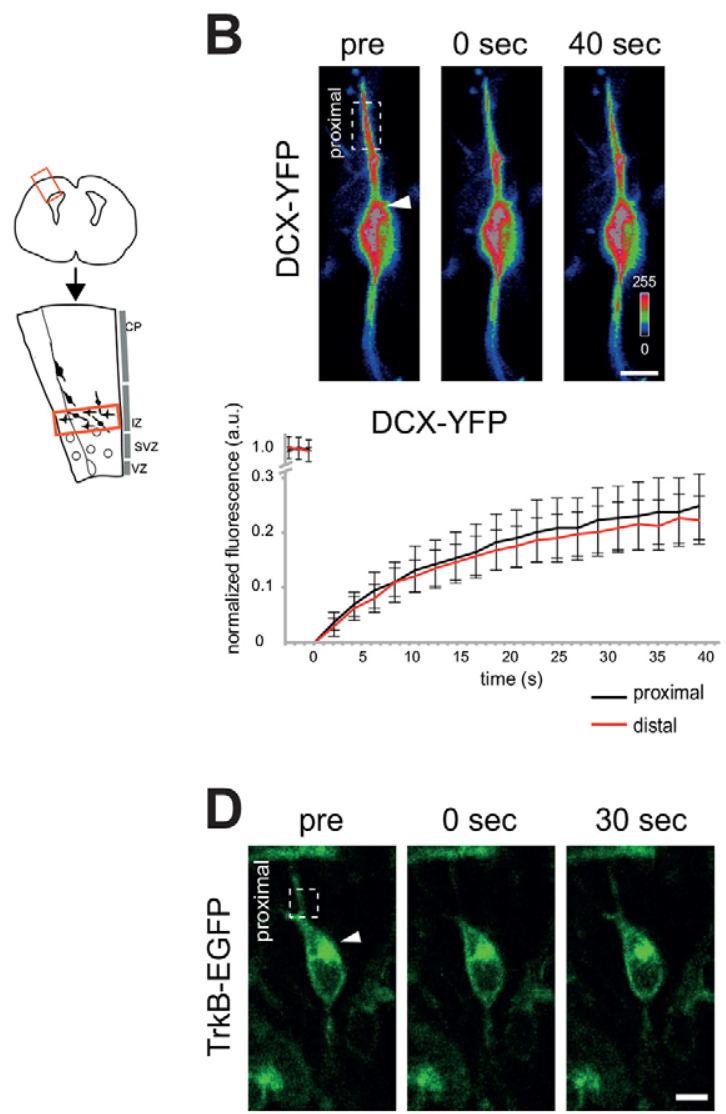
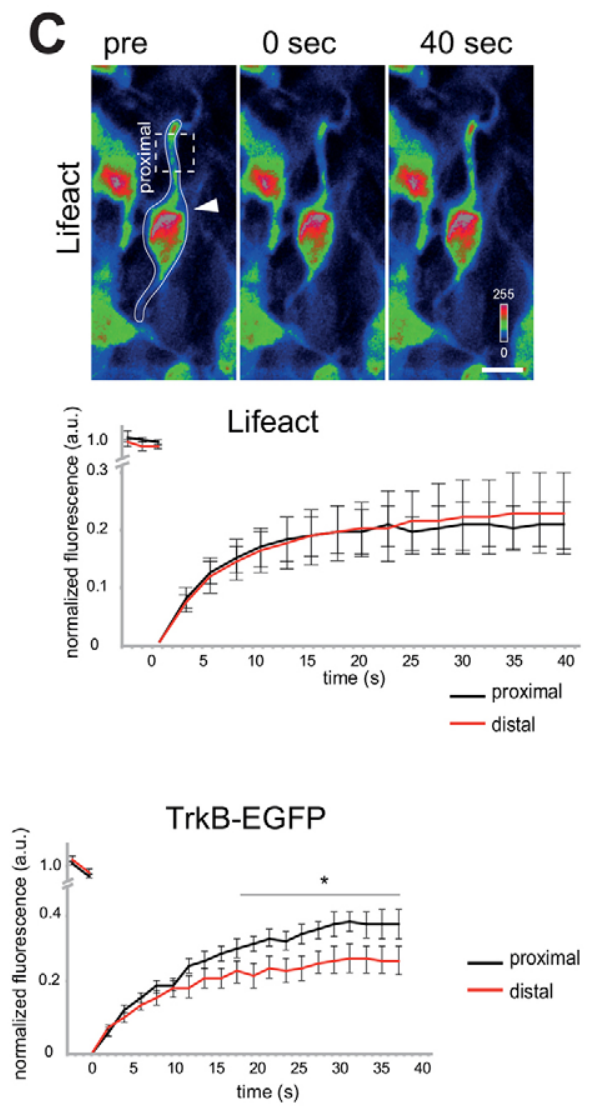

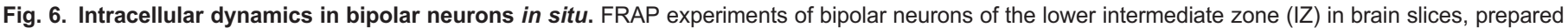
from E17 embryos which have been electroporated with the indicated GFP or YFP tagged proteins in utero at E15. Representative confocal pictures of the FRAP sequences are shown and below or beside the recovery curves. Upon bleaching of GFP- or YFP-tagged proteins within the proximal and/or the distal neurite, fluorescence recovery was monitored for $40 \mathrm{~s}$. In these examples just the recovery of the proximal neurite is shown. Mean recovery curves ( \pm s.e.m.) are shown (for all conditions $>20$ cells from 3 independent experiments). (A) Schematic drawing of the area imaged. VZ, ventricular zone; SVZ,

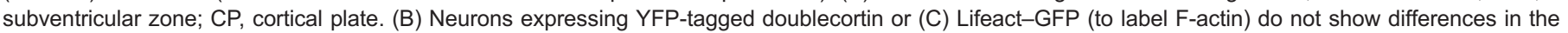
recovery between the two neurites, whereas in TrkB-GFP delivery into the proximal neurite is faster (D). ${ }^{*}<0.01$. Scale bars: $5 \mu$ m. White arrowheads,

Golgi position.

et al., 2014) and neurons that will shortly become multipolar (Tabata et al., 2009). We performed imaging of microtubule, actin and membrane dynamics in these neurons. In order to express GFP- or YFP-tagged proteins, cortical precursors were electroporated in utero in embryonic day (E)15 embryos with the respective constructs, and FRAP analysis was carried out in bipolar neurons in the intermediate zone of cortical slices from E17 embryos. We measured recovery rates of DCX-YFP, which revealed that in the more complex environment of the brain MT dynamics is also not polarized in any of the two opposite neurites of bipolar neurons (Fig. 6B). The same was true for actin turnover, which was analysed using the LifeAct-GFP construct which labels F-actin (Riedl et al., 2008). Fig. 6C shows that in situ actin turnover is also similar in the two neurites of bipolar neurons. Finally, in utero transfection of E15 rat embryos with the TrkB-GFP plasmid confirmed the preferential transport of the receptor towards the Golgi-facing neurite in bipolar neurons located in the lower intermediate zone (Fig. 6D).

\section{The upstream mechanism of membrane polarization in bipolar neurons}

It is known that developing neurons in vitro respond to polarized gradients of BDNF with axon outgrowth (Shelly et al., 2007). Our observation that the traffic of both TfR and the BDNF receptor TrkB is higher towards one neurite in bipolar neurons (Figs 5A,E; Fig. 6D) suggests that polarized growth might be induced by a gradient of BDNF. Thus, the simple reflection of bulk polarized membrane flow might determine later polarity. However, it is difficult to argue that a gradient of BDNF triggers higher receptor trafficking as in vitro ligands are uniformly distributed. The question is then what determines the polarization of membrane carriers. One possibility is that directional membrane trafficking is driven by the continuous polarized signalling of N-cadherin, which we showed correlates with the first polarization of neurons (see Fig. 3). To address this we analysed the distribution of the endogenous $\mathrm{N}$-cadherin with an antibody that recognizes an extracellular epitope of $\mathrm{N}$-cadherin. This analysis revealed that $\mathrm{N}-$ cadherin is mostly enriched in one of the neurites of bipolar neurons, being distributed along the shaft and at the tip of the neurite (Fig. 7A). To determine whether $\mathrm{N}$-cadherin might exert a role as an asymmetric membrane transport determinant we exposed one of the two neurites of bipolar neurons to exogenous $\mathrm{N}$-cadherin. This was accomplished by seeding hippocampal neurons on coverslips coated with alternating stripes of $\mathrm{N}$ cadherin or PLL. Time-lapse microscopy of bipolar neurons with the cell body and both neurites on PLL revealed that as soon as 

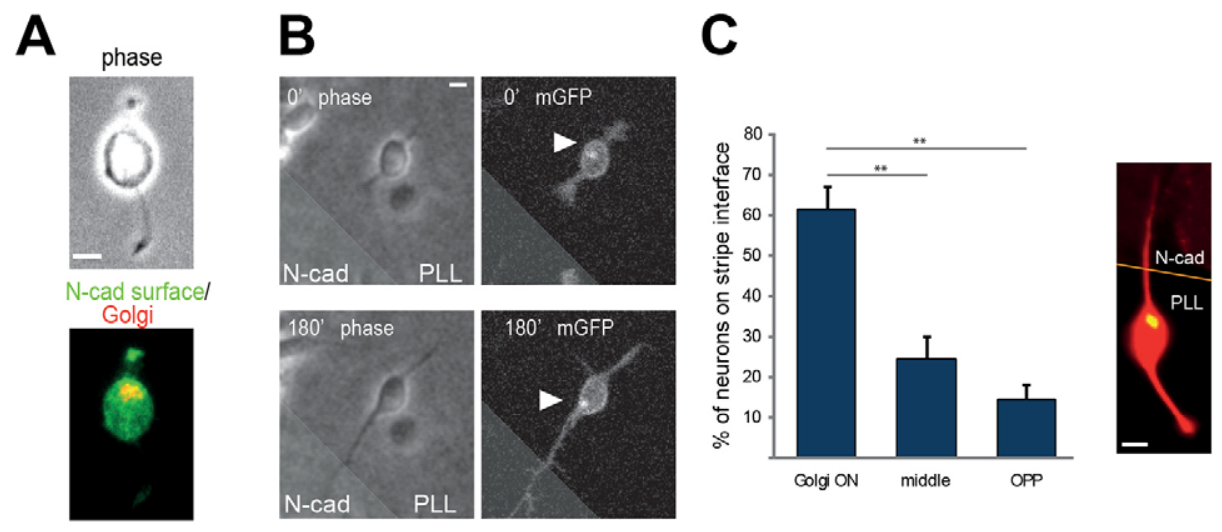

Fig. 7. Endogenous $\mathrm{N}$-cadherin is polarized in bipolar neurons. (A) Phase-contrast imaging of bipolar neurons and immunofluorescence labelling of

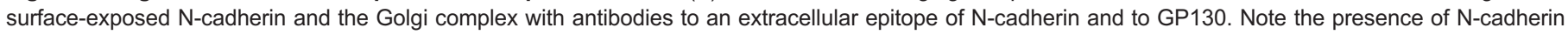
accumulation close to the Golgi-proximal neurite (49 cells analysed from three different experiments, 29 cells have the N-cadherin polarized towards the Golgi side, 10 to the opposite side and 10 have a diffuse distribution). (B) Time-lapse sequence of a bipolar neuron transfected with membrane-bound GFP and developing on PLL close to the interface between N-cadherin and PLL during the contact with N-cadherin. The Golgi complex (white arrowhead) is

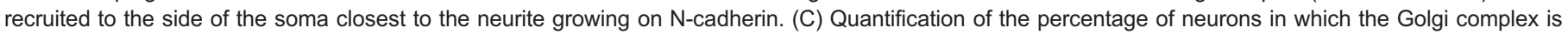
located close to the neurite contacting N-cadherin (Golgi ON), in the middle portion of the soma (middle) or on the opposite side of the soma (OPP). Only

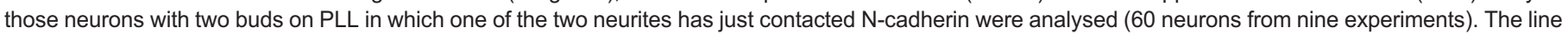
in the image marks the border between N-cadherin and PLL. Scale bars: $5 \mu \mathrm{m}$.

one of the two growing neurites contacts the N-cadherin stripe, the Golgi moves in the direction of this neurite and that this neurite later undergoes further growth (Fig. 7B). For quantitative assessment of the phenomenon, we considered the number of bipolar neurons whose cell body was far away from the $\mathrm{N}$ cadherin stripe and contacted the N-cadherin stripe just after becoming bipolar. In $61 \%$ of those neurons the Golgi was located at the N-cadherin-contacting pole (Fig. 7C), corroborating the hypothesis that $\mathrm{N}$-cadherin polarization might suffice to induce not only the formation of the first asymmetry of the round cell but also the preferential growth in the bipolar stage.

\section{DISCUSSION}

In this work, we show that the essential processes of the growth machinery of cells, including microtubule and actin dynamics and membrane trafficking, are strongly polarized before morphological polarity occurs (Figs 1, 2). We demonstrate that polarized microtubule growth and membrane trafficking change their location with time and become restricted to the place of morphological asymmetry only after the first neurite emerges (Fig. 2E,F). In contrast, the polarization of actin filament growth appears to act as a signpost for the site of formation of the first neurite and where MT and membrane polarization later become oriented, showing that the organization of actin is upstream of polarized microtubule growth and membrane trafficking. Thus, polarized distribution of intracellular components can be identified in both the round and bipolar cell stages. However, the polarized distribution has distinct temporal hierarchies: polarized actin dynamics marks the site of first polarization in round cells, whereas polarized membrane dynamics precedes asymmetric growth in the bipolar stage.

We have previously shown that polarization of N-cadherin in the immediate post-mitotic stage is an early and crucial mechanism in neuronal polarity. Here, we have shown that $\mathrm{N}$-cadherin colocalizes with the F-actin accumulation (Fig. 3A,B), and we suggest that the polarized actin dynamics observed in round neurons is the consequence of the N-cadherin 'cap'. This notion is supported by previous work from our laboratory: (1) a cadherin crescent becomes visible in Drosophila sensory neurons within $2 \mathrm{~min}$ after the last neurogenic progenitor division (Pollarolo et al., 2011); (2) endogenous N-cadherin is polarized in very young round neurons in the subventricular and intermediate zones of the mouse embryonic cortex (Gärtner et al., 2012), indicating that it is also of relevance in vivo; and (3) chromophore-assisted laser inactivation of GFP-cadherin at the site of the future first neurite prevents the formation of the first sprout indicating that cadherin clustering is an upstream event (Pollarolo et al., 2011). In Drosophila neurons, cadherin accumulation seems to be defined by the local clustering and recruitment of phosphatidylinositol 4,5-bisphosphate [PI $(4,5)$ $\mathrm{P}_{2}$ ], RhoA and Aurora kinase, in turn with polarized distribution inherited from the cleavage furrow of the last division (Pollarolo et al., 2011). It is well known that cadherin signalling affects actin cytoskeleton dynamics (Bard et al., 2008; Ouyang et al., 2013) and that a change in actin dynamics is sufficient to elicit neurite growth (Bradke and Dotti, 1999). In this context, the polarization of membrane trafficking and MT polymerization in the direction of the newly formed neurite would then serve to stabilize the new sprout. Whereas polarization mediated by cell-cell signalling could explain the polarization of all neuronal types, different types of neurons might utilize different adhesive or diffusible cell-cell molecular determinants. Future work in different neuronal populations will elucidate this aspect.

A second important finding was that differences in cytoskeletal turnover are not required for the subsequent development of morphological asymmetry: in bipolar neurons, the polarization of actin cytoskeleton dynamics or MT dynamics (Fig. 4), does not mark the future axon. Taking into account that MT dynamics is polarized in neurons in vitro during (and just shortly before) axon outgrowth, and that actin dynamics is polarized in neurons with several neurites and during neuronal migration (Bradke and Dotti, 1999; Sakakibara et al., 2014; Solecki et al., 2009; Witte et al., 2008) our new results suggest that polarized actin and MT dynamics, albeit present in the earliest stages, do not contribute to the polarity 'memory' at the bipolar stage, even though one of the two neurites is in the process of becoming distinct from the other, in morphology and dynamics. 
Interestingly, we found that membrane turnover, in terms of both exocytosis and endocytosis, is asymmetrically distributed in bipolar cells (Fig. 5). One can envision that the existence of a polarized, larger pool of membrane in one neurite serves the function of maintaining the optimal density of molecular complexes and receptors that can guarantee polarized growth even under the absence of polarized external cues. Upstream of the polarized membrane flow appears to be polarized N-cadherin. In fact, the encounter of a growth cone of a bipolar neuron with an $\mathrm{N}$-cadherin-rich environment induces the translocation of the Golgi to the pole of the soma facing the base of the N-cadherincontacting neurite and its faster growth. This observation is consistent with the hypothesis that membrane proteins required for polarity 'memory' and/or 'consolidation' (or otherwise important for later steps in axon specification) are maintained in the Golgi-proximal neurite by local membrane recycling, which would minimize the fast lateral diffusion and prepare this site for outgrowth. Restriction to this neurite occurs in turn by an $\mathrm{N}$-cadherin- and actin-mediated signalling. Alternatively, this restriction in membrane delivery could also be the cause of the accumulation of $\mathrm{N}$-cadherin at this location or be a component of a positive feed-forward process. Indeed, in bipolar neurons, in spite of active membrane trafficking, there is no net neurite outgrowth (for a considerable time supplementary material Fig. S3) suggesting that exocytotic and endocytotic events are balanced and allow very limited membrane outgrowth and retraction.

We found a similar intracellular organization of bipolar, notmigrating neurons in the in situ context (Fig. 6; supplementary material Fig. S4). Even though the bipolar stage in situ does not necessarily correspond to the exact same developmental stages in vitro, our findings suggest that polarity mechanisms found in dissociated neurons have relevant parallels in vivo. In the future, it will be interesting to combine the analysis of intracellular organization using tagged marker proteins with long-term timelapse imaging in order to unravel changes in intracellular organization throughout all developmental stages in vivo. In vivo, it has been shown that excitatory cortical neurons form their axon either during their multipolar stage (de Anda et al., 2010; Hatanaka and Yamauchi, 2013; Namba et al., 2014; Sakakibara et al., 2014) or during their bipolar migrating phase (Noctor et al., 2004; Sakakibara et al., 2014). It thus seems that neurons can establish axonal polarity at different times, perhaps depending on their origin. In fact, Tabata and colleagues (Tabata et al., 2009) have shown that the migratory behaviour of neurons derived from either radial glia progenitors or intermediate progenitors differ. Interestingly, centrosome position in vivo is correlated with the future axon (Andersen and Halloran, 2012; de Anda et al., 2010) or with the 'dominant' process (Sakakibara et al., 2014) in neurons that form their axon during the multipolar stage. By contrast, in bipolar neurons, the axon forms on the opposite side of the centrosome, which is located at the base of the leading neurite (Sakakibara et al., 2014). This is an interesting correlation to the in vitro situation in which the centrosome is localized at the base of the future axon or opposite to it (de Anda et al., 2008).

In summary, our work shows that a stereotyped sequence of events orchestrates the development of morphological polarity in neurons. In round neurons, before any neurite is formed, a localized cell-cell signalling machinery determines the creation of patches of highly dynamic actin. These in turn determine a change in membrane organization leading to the appearance of a first sprout followed by the movement of the Golgi and centrosomes to this site, with the consequent polarized movement of microtubules and of membrane cargoes. In bipolar neurons, membrane turnover is polarized to the future axon. One of the mechanisms involved in the maintenance of polarized membrane delivery to the first neurite seems to be the result of polarized $\mathrm{N}$-cadherin signalling. This sequence of events is likely to be instrumental for the correct wiring and positioning of neurons in the developing brain.

\section{MATERIALS AND METHODS}

\section{Constructs}

The vector encoding GFP-Rab5 was a gift from Marino Zerial (Max Planck Institute of Molecular Cell Biology and Genetics, Dresden, Germany). TfR-GFP was provided by Gary Banker (Oregon Health Sciences University, Portland, OR). The EB3-GFP vector was provided by Niels Galjart (Erasmus University, Rotterdam, The Netherlands). Utr-GFP was provided by William M. Bement (University of Wisconsin-Madison, Madison, WI). For the preparation of Utr-GFP under the CAG promoter, a BamHI/NotI fragment was cloned into a custom-made pCAGEN-MCS vector. $\alpha$-Tubulin-EGFP was obtained from Clontech (CA, USA). The TrkB-EGFP construct with the CMV promoter was purchased from Addgene (MA, USA) and an EcoRI/NotI fragment containing TrkB-EGFP was cloned under the CAG promoter of the pCAGEN vector (Addgene). Doublecortin-YFP CMV was provided by Reinhard Koester (University Braunschweig, Germany) and a doublecortin-YFP AgeI/NotI fragment was cloned into the pCAG-YFP vector (Addgene). Lifeact-EGFP-CMV was provided from Frank Bradke (University Bonn, Germany) and cloned under the pCAG promoter using EcoRI and NotI. The Golgi was fluorescently marked by tagging a Golgi localization sequence [the Nterminal 81 amino acids of human galactosyltransferase (Llopis et al., 1998)] at the N-terminus with Tag-RFP (kind gift of Oliver Griesbeck, Max Planck Institute of Neurobiology, Martinsried, Germany) or by using a palmitoylated version of EGFP, labelling intra- and extra-cellular membranes including the Golgi (supplementary material Fig. S1A). The palmitoylated EGFP is an EGFP N-terminally tagged by the first 20 amino acids of GAP-43 expressed under the control of the chicken actin promoter. The YFP-p58 plasmid was kindly provided by Ragna Sannerud (Sannerud et al., 2006).

\section{Primary cultures}

Rat embryonic hippocampal neurons were prepared as previously described (Banker and Goslin, 1988) and plated at a density of 2500 cells per $\mathrm{cm}^{2}$ on poly-L-lysine (PLL)-coated coverslips. Neurons were transfected in suspension using nucleofection (Amaxa). Transfected neurons were kept in suspension before plating for $2 \mathrm{~h}$ to allow the expression of the transgene and, at the same time, avoid the initiation of differentiation. This procedure has the additional advantage to provide time for the regeneration of surface molecules ( $\mathrm{N}$-cadherin, integrins etc.), which might have been shed by the trypsin treatment used for dissociation of the neuronal tissue (Takeichi and Nakagawa, 2001). Neurons were transfected in suspension using nucleofection (Amaxa).

\section{Culturing of neurons on patterned substrata}

Coverslips with alternating stripes of $\mathrm{N}$-cadherin $(10 \mathrm{mg} / \mathrm{ml}, \mathrm{R} \& D$ Systems, N-terminal extracellular domain) and PLL were prepared using silicon matrices (provided by Susanne Lang, MPI for Developmental Biology, Tübingen). PPL-coated coverslips were placed on top of the dry matrices and the channels filled with the substrates and an Alexa-Fluor-568-labelled secondary antibody in PBS. After $2 \mathrm{~h}$ incubation at $37^{\circ} \mathrm{C}$, channels were washed with PBS, coverslips were removed from the matrix and neurons were plated immediately. The development of individual neurons was observed on CELLocate coverslips (Eppendorf) or by time-lapse microscopy.

\section{Bead coating}

Polybead ${ }^{\circledR}$ microspheres (diameter, $1 \mu \mathrm{m}$; Polysciences), were precoated for $1 \mathrm{~h}$ at $37^{\circ} \mathrm{C}$ with PLL and washed. Beads were incubated 
with anti-human-Ig Alexa-Fluor-568-labelled antibody in combination with the N-cadherin N-terminal extracellular domain $(10 \mathrm{mg} / \mathrm{ml}, \mathrm{R} \& \mathrm{D}$ Systems) or with tenascin C (25 mg/ml; Chemicon). Following passive adsorption, microspheres were washed in medium and added to freshly plated neurons.

\section{In utero electroporation}

All animal experiments were approved by the Ethics Committee of the K. U. Leuven. Plasmids were injected in the lateral ventricles of E15 mouse embryos. Pregnant Swiss mice (E 14.5) were anesthetized by intramuscular injections of $88 \mathrm{mg}$ ketamine and $132 \mathrm{mg}$ xylazine per gram of body weight, uterine horns were exposed and the plasmids (1$2 \mu \mathrm{g} / \mu \mathrm{l}$ ) mixed with Fast Green (Sigma) were microinjected in the lateral ventricles of embryos. Five current pulses ( $50 \mathrm{~ms}$ pulse $/ 950 \mathrm{~ms}$ interval) were delivered across the head of the embryos ( $36 \mathrm{~V}$ ) targeting the dorsal-medial part of the cortex. After 2 or 3 days, mother mice were killed and embryonic brains were cut coronally with the vibratome $(250 \mu \mathrm{m})$ and cultured until live imaging was performed.

\section{Live-cell imaging, FRAP and fluorescence microscopy analysis}

Neurons were transferred to PLL-coated coverslips and imaged at high frequency in closed stainless steel chambers. Slice cultures were attached to a glass coverslip with Matrigel (BD biosciences) and imaged. The chambers were tightly closed to avoid $\mathrm{pH}$ changes. Neuronal development and the distribution of intracellular organelles and fluorescent beads were observed in time frames of 2-10 min by phase contrast or epifluorescence, using the perfect focus system of a Nikon confocal microscope equipped with a heated stage. Alternatively epifluorescent images were taken using a CellR Olympus microscope equipped with a heated stage. In some experiments the Golgi was labelled with WGA-Alexa-Fluor-647. For this, neurons were incubated with $2.5 \mu \mathrm{g} / \mathrm{ml} \mathrm{WGA}$ for $30 \mathrm{~min}$, washed with pre-equilibrated medium and imaged.

For FRAP experiments, three to five pre-bleaching images were acquired for the baseline. Bleaching was performed with 2 to 5 pulses on designed areas using the $488-\mathrm{nm}$ laser line of an argon laser (100 $\mathrm{mW}$ nominal output) at $90 \%$ power. If bleaching was not sufficient the 514-nm laser line was also used. Fluorescence recovery was monitored by collecting 30-120 single-section images at 1 - or 2-s intervals with low laser intensity ( $2 \%$ of intensity with the single 488 $\mathrm{nm}$ laser line, detection at $520-650 \mathrm{~nm}$ ). For quantitative analysis of fluorescence recovery and bleaching correction, data were normalized as previously described (Phair et al., 2004). Briefly, three regions of interest (ROIs) were determined: the bleached area, the whole cell and the background. At each time point, the average fluorescence intensity of the background was subtracted from the average fluorescence in the bleached area and in the whole cell. The background-corrected intensity of the bleached area was divided by the fluorescence intensity of the whole cell at each time point and normalized to the pre-bleaching average.

For the analysis of fluorescence signal along the periphery of cells (Factin, Sec8 and endosomes) the distribution of fluorescence was measured with a custom-made Image J routine based on the 'oval profile' macro (http://rsb.info.nih.gov/ij/plugins/oval-profile.html). Briefly, the perimeter of the cell was manually selected, the fluorescence signal and the position of the bead or the Golgi were determined with the oval profile macro. Signals were automatically aligned in Microsoft Excel to the centre of the bead (or Golgi), normalized to their maximum intensity and averaged. The manual tracking tool of Image J was used for tracking EB3 comets and TfR carriers.

Relative membrane supply (as labelled by palmitoylated GFP, p58, TfR and TrkB) was calculated as the number of organelles (with a diameter of $>0.5 \mu \mathrm{m}$ ) transported in a 30 -s time frame in either in the distal or the proximal neurite. To facilitate the count of newly-transported organelles, neurites were bleached. The values are expressed as a fraction of the total number of transported organelles in both neurites. Images were analysed with Image $\mathrm{J}$ : following automatic thresholding, the number of organelles was determined with the inbuilt Image J 'Particle Analyzer'.

\section{FM4-64 uptake in neurons}

Hippocampal neurons were incubated with FM4-64FX $(10 \mu \mathrm{M}$, Invitrogen) for $2 \mathrm{~min}$ in $\mathrm{N} 2$ medium at $37^{\circ} \mathrm{C}$ and under $5 \% \mathrm{CO}_{2}$; then cells were washed three times with cold $\mathrm{N} 2$ medium and quickly fixed at room temperature in 4\% PFA for 4 min. In order to quantify the FM4-64 signal, images were acquired at constant parameters of illumination and gain.

\section{Immunocytochemistry}

Neurons were fixed with 4\% PFA (supplemented with $1.44 \mathrm{M}$ sucrose, $1 \mathrm{M} \mathrm{MgCl}_{2}$ and $100 \mathrm{mM} \mathrm{EGTA}$ ) at $37^{\circ} \mathrm{C}$ for $10 \mathrm{~min}$ or for $3 \mathrm{~min}$ followed by post-fixation for $3 \mathrm{~min}$ in methanol at $-20^{\circ} \mathrm{C}$ for cytoskeletal stainings. Cells were permeabilized for $3 \mathrm{~min}$ in $0.1 \%$ Triton X-100 in PBS. After blocking in $2 \% \mathrm{FBS}, 2 \% \mathrm{BSA}$, and $0.2 \%$ fish gelatine in PBS, neurons were incubated with primary antibodies for either $1 \mathrm{~h}$ at room temperature or at $4^{\circ} \mathrm{C}$ overnight. After washing in PBS, secondary Alexa-Fluorconjugated antibodies (Invitrogen) were added for $45 \mathrm{~min}$ before embedding and imaging. The following primary antibodies were used: anti-pericentrin and anti- $\beta$ III-tubulin (Covance); anti-GM130, anti- $\gamma$ tubulin, anti- $\beta$ III-tubulin (Sigma); anti- $\alpha$-tubulin (Calbiochem); anti-tau1 (Chemicon); anti-phosphorylated-tyrosine (Upstate); anti-Sec8 (a kind gift by Shu-Chan Hsu, Rutgers University, NJ). Nuclei were visualized using Hoechst 33342 (Invitrogen). N-cadherin on the surface of neurons was detected by incubating neurons after fixation without permeabilization with an antibody recognizing a surface epitope of N-cadherin (clone GC-4, Sigma). Vibratome $100-\mu \mathrm{m}$ sections of embryonic brains were permeabilized and blocked at $4^{\circ} \mathrm{C}$ for $2 \mathrm{~h}$ in PBS with $0.3 \%$ Triton $\mathrm{X}$ $100,3 \%$ BSA and $3 \%$ goat serum, incubated with the primary antibody (rb Tbr2, Abcam) at $4{ }^{\circ} \mathrm{C}$ overnight and the secondary Alexa-Fluor-conjugated antibodies for $3 \mathrm{~h}$ at room temperature.

\section{Acknowledgements}

We thank Krist'l Vennekens for technical help and Sebastian Munck for help in imaging experiments.

\section{Competing interests}

The authors declare no competing interests.

\section{Author contributions}

The overall study was conceived and supervised by C.G.D. and A.G. A.G. and E.F.F. designed, performed and analyzed all experiments. A.G., E.F.F., F.V. and C.G.D. wrote the manuscript.

\section{Funding}

This work was partially supported by the Flanders Fund for Scientific Research [grant number FWO G 0.666.10N], NEUROBRAINNET grant from the Belgian Government [grant number IAP 7/16]; a Flemish Government Methusalem Grant; the Spanish Ministry of Science [grant number SAF2010-14906]; and Innovation Ingenio-Consolider [grant number CSD2010-00045] to A.G. and C.G.D. The support of the MIUR-PRIN Program to F.V. and E.F.F. is also acknowledged. E.F.F. has been in part supported by an EMBO fellowship [grant number ASTF: 369.00-2007]. The Nikon confocal microscope was acquired through a Hercules Type 1 grant [grant number: AKUL/09/037].

\section{Supplementary material}

Supplementary material available online at

http://jcs.biologists.org/lookup/suppl/doi:10.1242/jcs.149815/-/DC1

\section{References}

Andersen, E. F. and Halloran, M. C. (2012). Centrosome movements in vivo correlate with specific neurite formation downstream of LIM homeodomain transcription factor activity. Development 139, 3590-3599.

Attardo, A., Calegari, F., Haubensak, W., Wilsch-Bräuninger, M. and Huttner, W. B. (2008). Live imaging at the onset of cortical neurogenesis reveals differential appearance of the neuronal phenotype in apical versus basal progenitor progeny. PLOS ONE 3, e2388.

Banker, G. and Goslin, K. (1988). Developments in neuronal cell culture. Nature 336, 185-186.

Bard, L., Boscher, C., Lambert, M., Mège, R. M., Choquet, D. and Thoumine, O. (2008). A molecular clutch between the actin flow and N-cadherin adhesions drives growth cone migration. J. Neurosci. 28, 5879-5890.

Betz, W. J. and Bewick, G. S. (1992). Optical analysis of synaptic vesicle recycling at the frog neuromuscular junction. Science 255, 200-203. 
Bradke, F. and Dotti, C. G. (1997). Neuronal polarity: vectorial cytoplasmic flow precedes axon formation. Neuron 19, 1175-1186.

Bradke, F. and Dotti, C. G. (1998). Membrane traffic in polarized neurons Biochim. Biophys. Acta 1404, 245-258.

Bradke, F. and Dotti, C. G. (1999). The role of local actin instability in axon formation. Science 283, 1931-1934

Burack, M. A., Silverman, M. A. and Banker, G. (2000). The role of selective transport in neuronal protein sorting. Neuron 26, 465-472.

Burkel, B. M., von Dassow, G. and Bement, W. M. (2007). Versatile fluorescent probes for actin filaments based on the actin-binding domain of utrophin. Cell Motil. Cytoskeleton 64, 822-832.

de Anda, F. C., Gärtner, A., Tsai, L. H. and Dotti, C. G. (2008). Pyramidal neuron polarity axis is defined at the bipolar stage. J. Cell Sci. 121, 178-185.

de Anda, F. C., Pollarolo, G., Da Silva, J. S., Camoletto, P. G., Feiguin, F. and Dotti, C. G. (2005). Centrosome localization determines neuronal polarity. Nature 436, 704-708.

de Anda, F. C., Meletis, K., Ge, X., Rei, D. and Tsai, L. H. (2010). Centrosome motility is essential for initial axon formation in the neocortex. J. Neurosci. 30 10391-10406.

Dimidschstein, J., Passante, L., Dufour, A., van den Ameele, J., Tiberi, L., Hrechdakian, T., Adams, R., Klein, R., Lie, D. C., Jossin, Y. et al. (2013). Ephrin-B1 controls the columnar distribution of cortical pyramidal neurons by restricting their tangential migration. Neuron 79, 1123-1135.

Dotti, C. G., Sullivan, C. A. and Banker, G. A. (1988). The establishment of polarity by hippocampal neurons in culture. J. Neurosci. 8, 1454-1468.

Esch, T., Lemmon, V. and Banker, G. (1999). Local presentation of substrate molecules directs axon specification by cultured hippocampal neurons. J. Neurosci. 19, 6417-6426.

Esch, T., Lemmon, V. and Banker, G. (2000). Differential effects of NgCAM and $\mathrm{N}$-cadherin on the development of axons and dendrites by cultured hippocampal neurons. J. Neurocytol. 29, 215-223.

Flynn, K. C., Pak, C. W., Shaw, A. E., Bradke, F. and Bamburg, J. R. (2009) Growth cone-like waves transport actin and promote axonogenesis and neurite branching. Dev. Neurobiol. 69, 761-779.

Gärtner, A., Fornasiero, E. F., Munck, S., Vennekens, K., Seuntjens, E., Huttner, W. B., Valtorta, F. and Dotti, C. G. (2012). N-cadherin specifies first asymmetry in developing neurons. EMBO J. 31, 1893-1903.

Hatanaka, Y. and Murakami, F. (2002). In vitro analysis of the origin, migratory behavior, and maturation of cortical pyramidal cells. J. Comp. Neurol. 454, 1-14.

Hatanaka, Y. and Yamauchi, K. (2013). Excitatory cortical neurons with multipolar shape establish neuronal polarity by forming a tangentially oriented axon in the intermediate zone. Cereb. Cortex 23, 105-113.

Jossin, Y. and Cooper, J. A. (2011). Reelin, Rap1 and N-cadherin orient the migration of multipolar neurons in the developing neocortex. Nat. Neurosci. 14, 697-703.

Llopis, J., McCaffery, J. M., Miyawaki, A., Farquhar, M. G. and Tsien, R. Y. (1998). Measurement of cytosolic, mitochondrial, and Golgi pH in single living cells with green fluorescent proteins. Proc. Natl. Acad. Sci. USA 95, 68036808.

Menchón, S. A., Gärtner, A., Román, P. and Dotti, C. G. (2011). Neuronal (bi)polarity as a self-organized process enhanced by growing membrane. PLoS ONE 6, e24190.

Namba, T., Kibe, Y., Funahashi, Y., Nakamuta, S., Takano, T., Ueno, T. Shimada, A., Kozawa, S., Okamoto, M., Shimoda, Y. et al. (2014). Pioneering axons regulate neuronal polarization in the developing cerebral cortex. Neuron 81, 814-829.
Neukirchen, D. and Bradke, F. (2011). Cytoplasmic linker proteins regulate neuronal polarization through microtubule and growth cone dynamics. J. Neurosci. 31, 1528-1538.

Noctor, S. C., Martínez-Cerdeño, V., Ivic, L. and Kriegstein, A. R. (2004). Cortical neurons arise in symmetric and asymmetric division zones and migrate through specific phases. Nat. Neurosci. 7, 136-144.

Ouyang, M., Lu, S., Kim, T., Chen, C. E., Seong, J., Leckband, D. E., Wang, F., Reynolds, A. B., Schwartz, M. A. and Wang, Y. (2013). N-cadherin regulates spatially polarized signals through distinct p120ctn and $\beta$-catenin-dependent signalling pathways. Nat. Commun. 4, 1589.

Phair, R. D., Gorski, S. A. and Misteli, T. (2004). Measurement of dynamic protein binding to chromatin in vivo, using photobleaching microscopy. Methods Enzymol 375, 393-414.

Pollarolo, G., Schulz, J. G., Munck, S. and Dotti, C. G. (2011). Cytokinesis remnants define first neuronal asymmetry in vivo. Nat. Neurosci. 14, 1525-1533.

Polleux, F. and Snider, W. (2010). Initiating and growing an axon. Cold Spring Harb. Perspect. Biol. 2, a001925.

Polleux, F., Giger, R. J., Ginty, D. D., Kolodkin, A. L. and Ghosh, A. (1998). Patterning of cortical efferent projections by semaphorin-neuropilin interactions. Science 282, 1904-1906.

Polleux, F., Morrow, T. and Ghosh, A. (2000). Semaphorin 3A is a chemoattractant for cortical apical dendrites. Nature 404, 567-573.

Powell, S. K., Rivas, R. J., Rodriguez-Boulan, E. and Hatten, M. E. (1997). Development of polarity in cerebellar granule neurons. J. Neurobiol. 32, 223236.

Reiner, O. and Sapir, T. (2009). Polarity regulation in migrating neurons in the cortex. Mol. Neurobiol. 40, 1-14.

Riedl, J., Crevenna, A. H., Kessenbrock, K., Yu, J. H., Neukirchen, D., Bista, M., Bradke, F., Jenne, D., Holak, T. A., Werb, Z. et al. (2008). Lifeact: a versatile marker to visualize F-actin. Nat. Methods 5, 605-607.

Sakakibara, A., Sato, T., Ando, R., Noguchi, N., Masaoka, M. and Miyata, T. (2014). Dynamics of centrosome translocation and microtubule organization in neocortical neurons during distinct modes of polarization. Cereb. Cortex. 24, 1301-1310.

Sannerud, R., Marie, M., Nizak, C., Dale, H. A., Pernet-Gallay, K., Perez, F., Goud, B. and Saraste, J. (2006). Rab1 defines a novel pathway connecting the pre-Golgi intermediate compartment with the cell periphery. Mol. Biol. Cell 17, 1514-1526.

Shelly, M., Cancedda, L., Heilshorn, S., Sumbre, G. and Poo, M. M. (2007). LKB1/STRAD promotes axon initiation during neuronal polarization. Cell 129, 565-577.

Solecki, D. J., Trivedi, N., Govek, E. E., Kerekes, R. A., Gleason, S. S. and Hatten, M. E. (2009). Myosin II motors and F-actin dynamics drive the coordinated movement of the centrosome and soma during CNS glial-guided neuronal migration. Neuron 63, 63-80.

Tabata, H., Kanatani, S. and Nakajima, K. (2009). Differences of migratory behavior between direct progeny of apical progenitors and basal progenitors in the developing cerebral cortex. Cereb. Cortex 19, 2092-2105.

Takeichi, M. and Nakagawa, S. (2001). Cadherin-dependent cell-cell adhesion. Curr. Protoc. Cell Biol. Chapter 9, Unit 93.

Tissir, F. and Goffinet, A. M. (2003). Reelin and brain development. Nat. Rev. Neurosci. 4, 496-505

Wiggin, G. R., Fawcett, J. P. and Pawson, T. (2005). Polarity proteins in axon specification and synaptogenesis. Dev. Cell 8, 803-816.

Witte, H., Neukirchen, D. and Bradke, F. (2008). Microtubule stabilization specifies initial neuronal polarization. J. Cell Biol. 180, 619-632. 